\title{
Unsteady Flow Simulation of a Sweeping Jet Actuator Using a Lattice-Boltzmann Method
}

\author{
B. Duda* \\ Exa GmbH, Landshuter Allee 8, 80637 Munich, Germany \\ M. Wessels ${ }^{\dagger} \quad$ E. Fares ${ }^{\ddagger}$ \\ Exa GmbH, Curiestrasse 4, 70563 Stuttgart, Germany \\ V. Vatsa ${ }^{\S}$ \\ NASA Langley Research Center, Hampton, VA, 23681, USA
}

\begin{abstract}
Active flow control technology is increasingly used in aerospace applications to control flow separation and to improve aerodynamic performance. In this paper, PowerFLOW is used to simulate the flow through a sweeping jet actuator at two different pressure ratios. The lower pressure ratio leads to a high subsonic flow, whereas the high pressure ratio produces a choked flow condition. Comparison of numerical results with experimental data is shown, which includes qualitatively good agreement of pressure histories and spectra. PIV measurements are also available but the simulation overestimates mean and fluctuation quantities outside the actuator. If supply pressure is matched at one point inside the mixing chamber a good qualitative agreement is achieved at all other monitor points.
\end{abstract}

\section{Introduction}

Sweeping jet actuators are currently considered promising for active flow control applications on aircraft because of their simple and robust design as well as their usability over a wide range of flow conditions. Earlier experimental investigations ${ }^{1,2}$ have shown a complex dynamical behavior of the actuator, whose working principle is exemplified in figure 1. Flow passes through the main channel of the actuator and attaches to either one of the lateral walls due to the Coanda effect, which bends the jet outside the orifice into the opposite direction, see 1a). This leads to a reversed flow in the channel adjacent to the Coanda surface, which in return pushes the jet to the opposite side, see 1b). Finally, the jet reattaches on the opposite surface, bending the outside jet into the other direction, see 1c). The same feedback loop takes place and pushes the jet back into its original position. Previous numerical studies with PowerFLOW on this topic can be found for a single actuator configuration and for a high-lift configuration with 32 embedded actuators in $^{3}$ and in ${ }^{4}$, respectively.

Unsteady simulation techniques are required to properly predict the resulting flow field and therefore the Lattice-Boltzmann (LBM) solver PowerFLOW by Exa Corporation is chosen for this task. This paper is organized as follows: Section II explains the underlying numerical method of this simulation. The experimental test case is then described in III, whereas the numerical setup and results are given in section IV. Finally, conclusion and outlook are given in $\mathrm{V}$.

\footnotetext{
* Senior Application Engineer, Aerospace, AIAA member.

†Consultant, Aerospace Application.

${ }^{\ddagger}$ Senior Technical Director, Aerospace, Senior AIAA member.

$\S$ Senior Research Scientist, Associate Fellow, AIAA.
} 


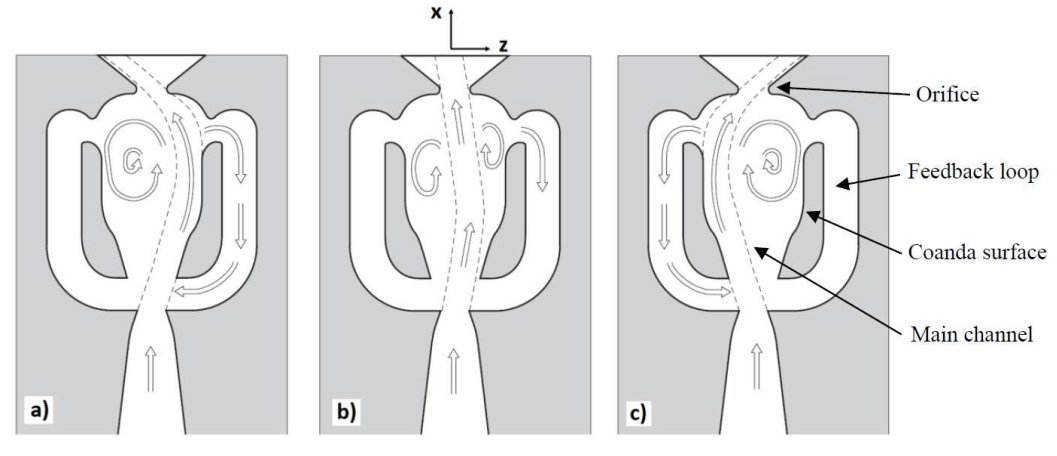

Figure 1. Working principle of sweeping jet actuator ${ }^{2}$

\section{Numerical Method}

LBM methods rely on solving a velocity distribution function on a Cartesian grid, which describes the local probability of particles travelling with a characteristic velocity into a discrete direction. This is in contrast to standard computational fluid dynamics (CFD) methods that solve a set of non-linear partial differential equations on a body-fitted mesh. In a three-dimensional discretization volume, referred to as a voxel, 19 velocity states per mesh node are allowed. This is known as the D3Q19 lattice scheme and it was shown by Chen et al. ${ }^{5}$ and Qian et al. ${ }^{6}$ that this is a sufficient discretization in order to recover the NavierStokes equations for an isotropic flow. Since transonic flow will also be encountered for some operating conditions of the actuator, the lattice scheme needs to be extended to capture this type of flow correctly. An overview of the necessary modifications are given by Fares et al. ${ }^{7}$ Once the distribution function is computed, macroscopic quantities like density and linear momentum are calculated by discrete summation. The remaining quantities are finally obtained by applying the thermodynamic relations of an ideal gas.

The underlying Cartesian discretization of the flow volume does not allow to resolve the boundary layer up to $y^{+} \approx 1$ for high Reynolds numbers because of the associated computational costs. Therefore, the boundary layers are modelled using a wall function. The turbulence modelling approach, called LBM-Very Large-Eddy Simulation (LBM-VLES), is incorporated in the solver PowerFLOW, which is used for this study. Lattice-Boltzmann methods are uniquely suitable for performing these type of simulation because they are by definition unsteady and have very low artificial diffusion. For a more detailed description on LBM-VLES the reader is referred to these publications. ${ }^{8-13}$ Additional validation studies performed with PowerFLOW can be found here. ${ }^{14-19}$

\section{Test Case}

The test case considered here is equivalent to the measurement campaign conducted by Kushner et al. ${ }^{1}$ In order to align the jet with the main flow, the actuator is contained in a fairing, which is mounted on the wind tunnel floor, cf. figure 2(a). Different pressure ratios have been investigated in combination with the wind tunnel main flow turned on and off. The numerical study in this paper is limited to two stagnation pressure levels supplied to the actuator, i.e., $p_{t} / p_{\infty}=1.9$ and 3.6. These ratios are chosen to represent a high subsonic and a transonic operating point of the actuator. In both cases, the wind tunnel main flow has a velocity of $56 \mathrm{~m} / \mathrm{s}$.

\section{Numerical Setup and Results}

A sketch of the computational domain is shown in figure 3. In order to minimize systematic errors, the test section of the wind tunnel is included into the simulation. The cross section has an area of $1219 \mathrm{~mm}$ $\mathrm{x} 813 \mathrm{~mm}$ and the measurement section has a length of $3048 \mathrm{~mm}$. The actuator has a height of $228.6 \mathrm{~mm}$ and is located $1321 \mathrm{~mm}$ downstream of the wind tunnel inlet. A block velocity profile with $U=56 \mathrm{~m} / \mathrm{s}$ is prescribed at the inlet and a pressure outlet with $p=101325 \mathrm{~Pa}$ is specified at the outflow plane. The wind tunnel walls are treated frictionless but all walls of the actuator, i.e., on the inside and on the outside, are 


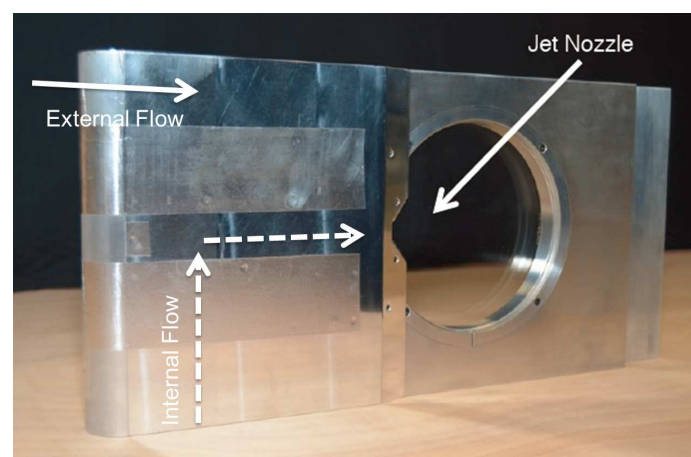

(a)

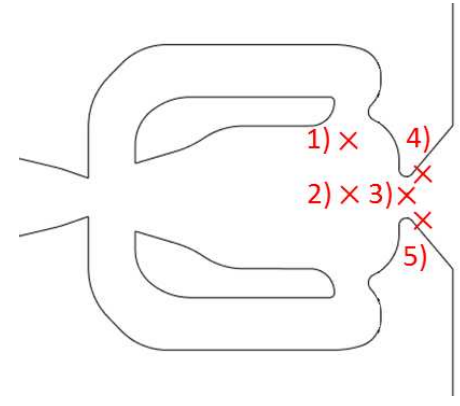

(b)

Figure 2. Wind tunnel model of faired actuator (a) and outline of internal flow geometry (b)

no-slip walls. An additional total pressure inlet is prescribed at the feeding pipe of the actuator. Ambient temperature is set to $T=297 \mathrm{~K}$ and viscosity for air is calculated according to Sutherland's law.

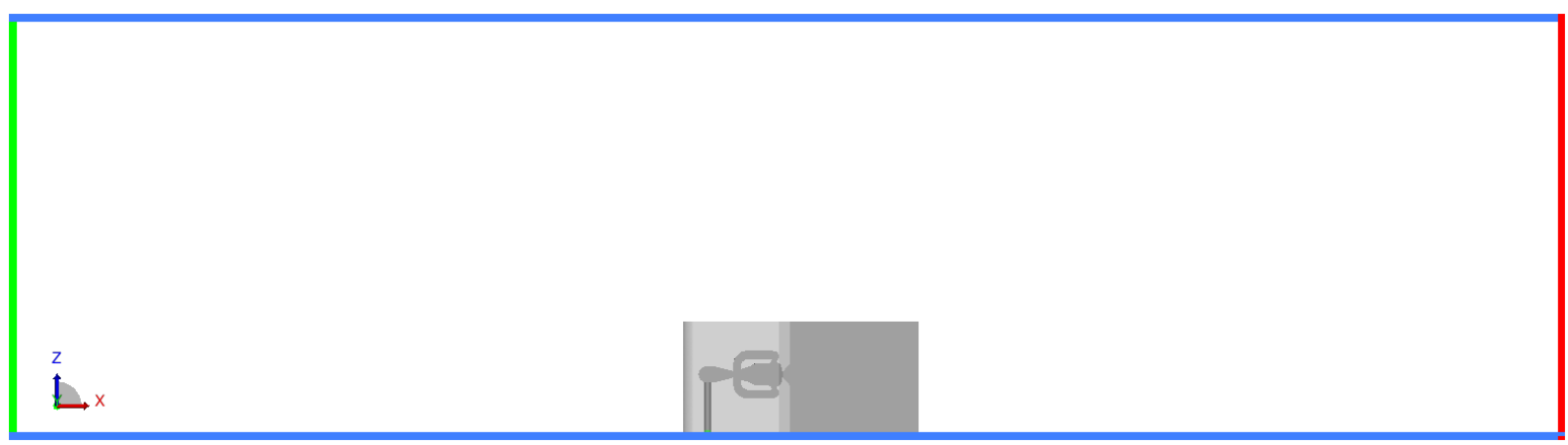

Figure 3. Computational Domain

Since spatial resolution requirements are different across the simulation domain, regions of variable resolution are introduced in the Cartesian lattice, which allow a doubling of the initial resolution in each direction. A grid resolution study is carried out with a refinement factor of 1.5 between two consecutive grid levels. The exit nozzle has an area of $6.35 \mathrm{~mm} \times 12.7 \mathrm{~mm}$ and is resolved by $5.33 \times 10.67$ voxels in the coarse, $8 \times$ 16 voxels in the medium and $12 \times 24$ in the fine case. The corresponding grid sizes for the entire domain are $2.3 \mathrm{M}, 7.2 \mathrm{M}$ and $22.8 \mathrm{M}$ voxels, respectively. Due to the numerical method, the physical time step is directly linked to the physical size of the smallest voxel, corresponding to a CFL number equal to 1 . The time steps are therefore $\Delta t=1.257 \mathrm{e}-06 \mathrm{~s}$ for the coarse, $\Delta t=8.383 \mathrm{e}-07 \mathrm{~s}$ for the medium and $\Delta t=5.589 \mathrm{e}-07 \mathrm{~s}$ for the fine resolution. The associated computational cost is given exemplary for the fine simulation: It amounts to 2,100 CPUh, which translates to 1.5 days run-time on 60 cores (Intel Xeon CPU E5-2640 0 @ 2.50GHz).

Pressure signals were recorded during the experiment at five different locations, cf. figure 2(b). The signals for two locations inside the actuator are shown in figure 4 for the high and low actuation pressure ratio and for all three mesh levels. For visibility, the graphs have been moved by a constant value of one for the medium and two for the fine resolution. All three mesh levels produce a very similar pressure evolution and hence show very little grid dependency. Observing more closely however, one can see that the mean levels are slightly higher for higher mesh resolution. This is consistent among all points shown and expected since higher grid resolution reduces numerical pressure losses.

A total time of $0.1 \mathrm{~s}$ has been simulated, which corresponds to roughly 12 characteristic times the external flow needs to pass over the actuator. After an initial transient time of $0.01 \mathrm{~s}$, time statistics have been collected. Figure 5 shows a snapshot of the Mach number contour on top and an averaged Mach number contour on the bottom for the fine resolution case on a plane through the actuator. The snapshot shows the state where the external jet is at its extreme deflection before moving into the opposite direction. The local Mach number reaches values close to 0.95 at the edges of the nozzle but the flow remains subsonic. The averaged Mach number contour shows a maximum at the nozzle but it does not exceed 0.84 . 
Concerning the higher pressure ratio, figure 6 shows the same quantities as above, i.e. instantaneous Mach number contour on top and an averaged Mach number contour on the bottom for the fine resolution case. Clearly, the higher pressure ratio leads to a transonic operating condition with a choked nozzle and a maximum average Mach number of 1.35. The overall extent of the supersonic flow area is rather small and does not extend more than 1.5 throat diameters downstream. The instantaneous view reveals a highest local Mach number of 1.74, again very close the corners of the throat. Supersonic pockets are visible as far as 5 throat diameters downstream. When comparing both operating conditions, the lateral spreading for the high actuation pressure is smaller than for the low actuation pressure.

Figures 7 and 8, show the pressure histories at all five experimental locations. A small offset is directly visible for the low actuation pressure whereas it is more pronounced in the high actuation case. Regarding shape and amplitude of the fluctuations however, an excellent agreement between simulation and experiment is visible for points 1,2 and 3, which all lie within the actuator. The behavior of points 4 and 5 is not as consistent but this aspect will be addressed by a location sensitivity study later on. Finally, these histories also justify the choice of $0.01 \mathrm{~s}$ initial transient time before time statistics are extracted.

The question remains why all pressure levels inside the actuator are underestimated compared to experiment. It is interesting to notice that the differences increase consistently when going from low to high actuation pressure. Figure 9 shows the averaged stagnation pressure distribution inside the actuator. There is only little pressure loss from the actuator inlet through the feeding pipe (not visible here, cf. figure 3) to the plenum on the far left even though the flow is bending by 90 degrees. This means that numerical losses can be excluded. The pressure drop inside the mixing chamber is also physical due to the expansion of the flow, the strong mixing and the back flow through the upper and lower feedback arms.

Since points 4 and 5 are positioned in an area with large flow gradients, a sensitivity study has been carried out for the fine resolution case with high actuation pressure. In addition to the location of the actual measurement point (referred to as center in the following), pressure signals were recorded on 4 points moved by $1.5 \mathrm{~mm}$ to the left, the right, the top and the bottom. Power spectral densities have been computed for these quantities and are shown in figure 10. Even though the high frequency content remains unaffected, large differences in the amplitudes of the four dominant peaks at 255, 515, 790 and $1050 \mathrm{~Hz}$ are visible. Only the bottom point for the low actuation pressure and the center point for the high actuation pressure are reproducing all four peaks. Compared to the experimental data, a shift of these peaks is observed to higher frequencies. The overall levels are overestimated in the simulations except for the first peak, which represent the sweeping motion of the jet.

As a final step, two additional simulations have been carried with adjusted boundary conditions at the actuator inlet. Following the preceding discussion about the differences observed in pressure levels, higher total pressure values have been supplied at the actuator inlet to approximately match the level at point 1 . In case of the low actuation pressure the pressure ratio was increased to 2.2 , whereas for the high actuation pressure it was increased to 5.1. Since mass flow should be kept constant, an isentropic compression was assumed and the inlet temperatures were therefore estimated to $373 \mathrm{~K}$ and $473 \mathrm{~K}$, respectively. Figures 11 and 12 show again the pressure histories for all 5 points for the low and high actuation pressure. Even though pressure was only matched at one point and the offset was not constant among all points, a considerable improvement is visible at all points.

Figure 13 shows the distribution of the mean $x$-velocity component (left) and the root mean square value of $x$-velocity (right) outside of the actuator for the high actuation pressure. The top image shows experimental PIV data, followed by three numerical results. The three numerical datasets are taken $2 \mathrm{~mm}$ below the center-plane, at the center-plane (as in experiment) and $2 \mathrm{~mm}$ above the center-plane of the nozzle. A high sensitivity on the plane location is visible as well as a large overestimation. This is in contrast to the pressure probes, where a qualitative good agreement is achieved. It remains interesting to notice that the velocity in experiments drops to the wind tunnel free stream velocity $\left(U_{\infty}=56 \mathrm{~m} / \mathrm{s}\right)$ extremely quickly. This is in contrast to the simulation where the influence of the actuator is still discernible in the far field. As this paper is written, there are still open questions about accuracy and quality of the PIV data.

\section{Conclusion and Outlook}

LBM based simulations of a sweeping jet actuator have been presented and compared to experimental data for two actuation pressures. As observed in experiments, the high actuation case produced a choked flow condition. Despite lower pressure levels predicted by the simulation, the pressure histories monitored 
inside the actuator agree very well with experimental data. For the probes located downstream of the throat differences between simulation and experiments become visible. This is attributed to the sensitivity of these signals on the probe location, which was shown by spectral analysis. The agreement of velocity contours outside of the actuator are not satisfactory yet and additional investigations, also on the experimental side, are needed. By adjusting the boundary conditions to match one point inside the mixing chamber, the prediction at all other locations could be improved considerably.

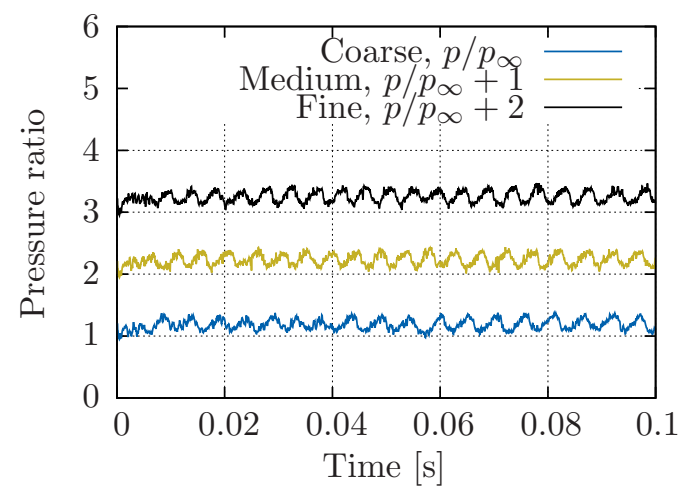

(a) Point 1, low actuation pressure

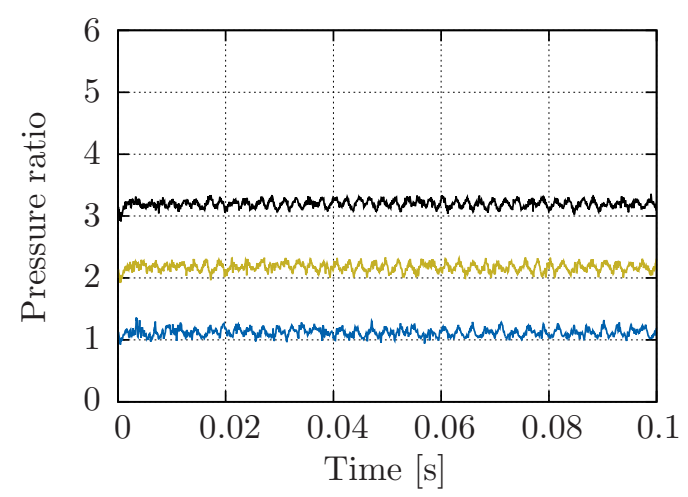

(c) Point 2, low actuation pressure

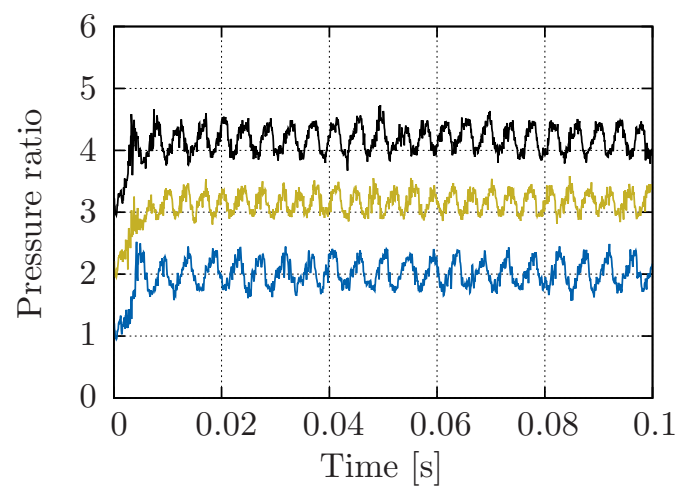

(b) Point 1, high actuation pressure

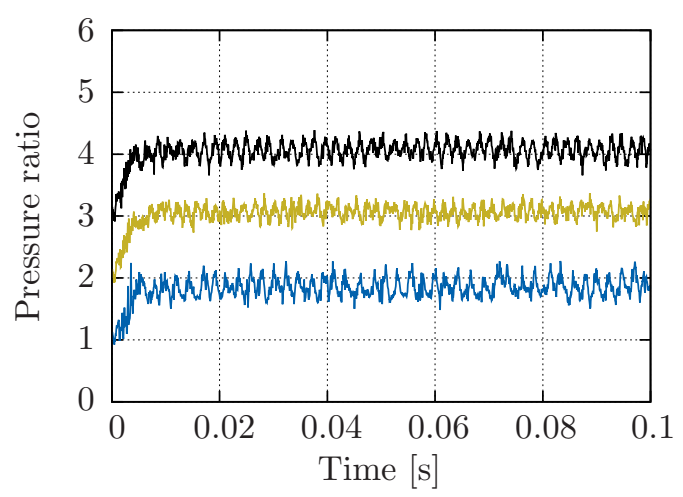

(d) Point 2, high actuation pressure

Figure 4. Pressure histories extracted for three different mesh resolutions 

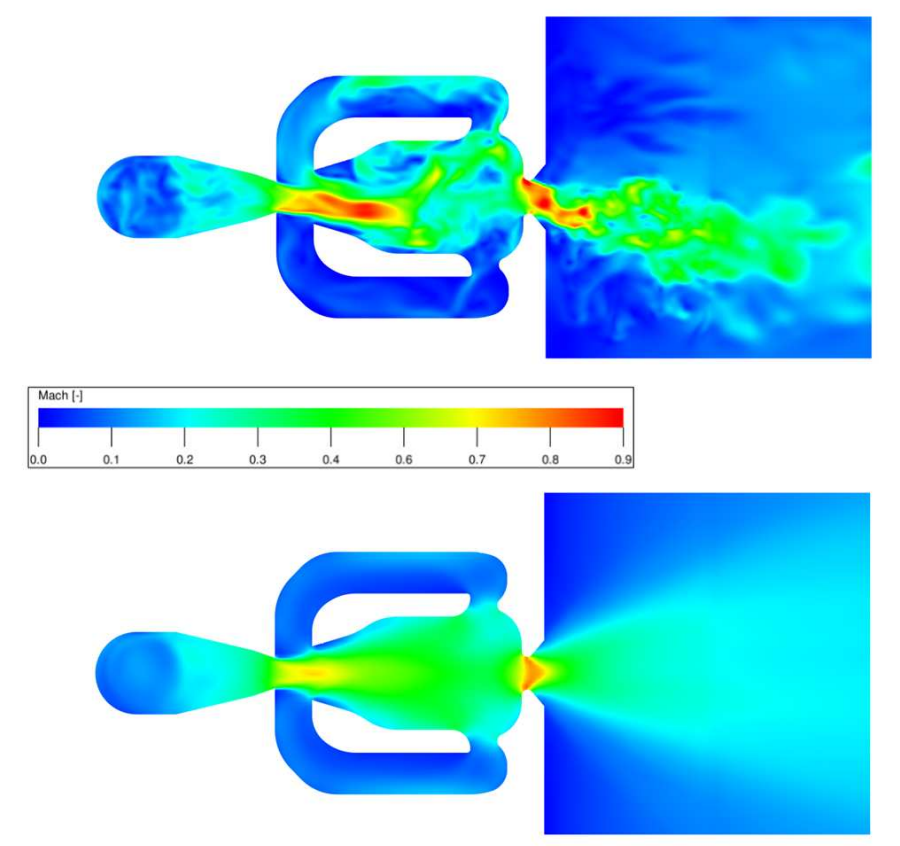

Figure 5. Snapshot (top) and averaged contour (bottom) of Mach number for low actuation pressure
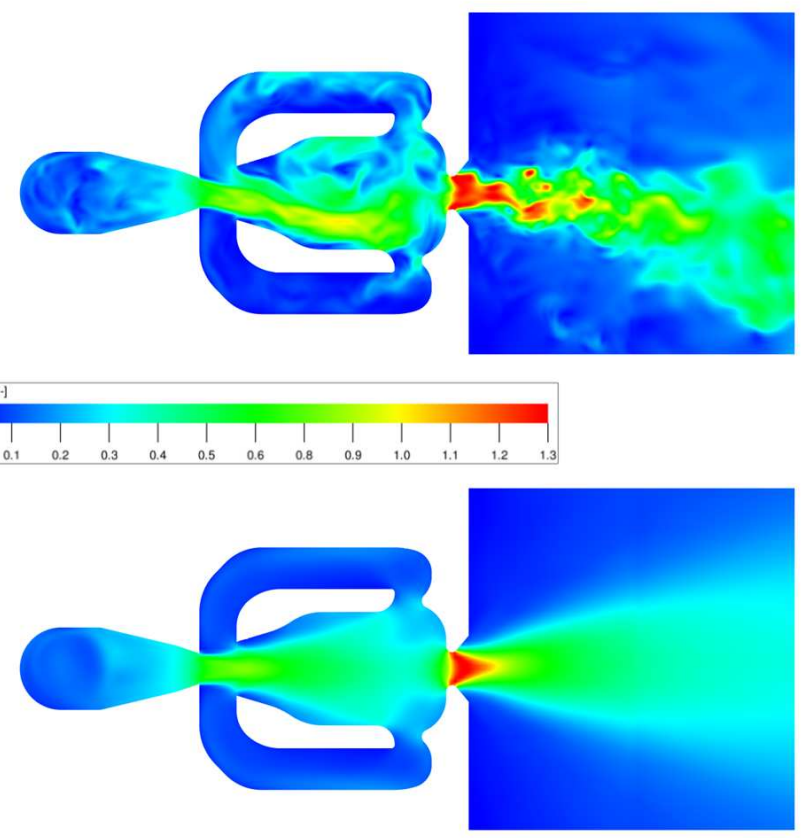

Figure 6. Snapshot (top) and averaged contour (bottom) of Mach number for high actuation pressure 


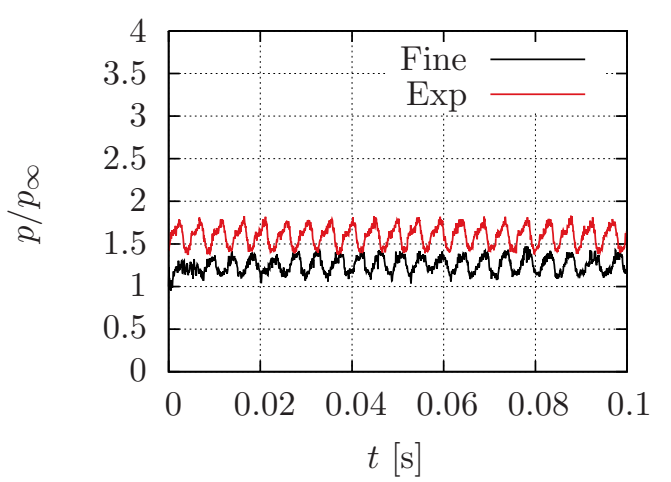

(a) Point 1, low actuation pressure

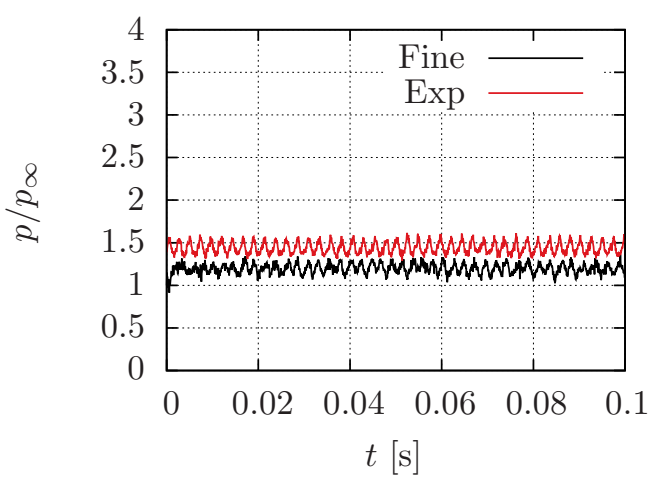

(c) Point 2, low actuation pressure

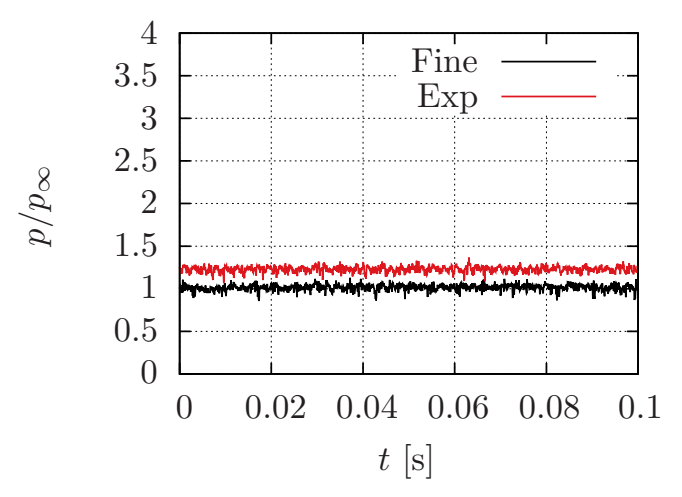

(e) Point 3, low actuation pressure

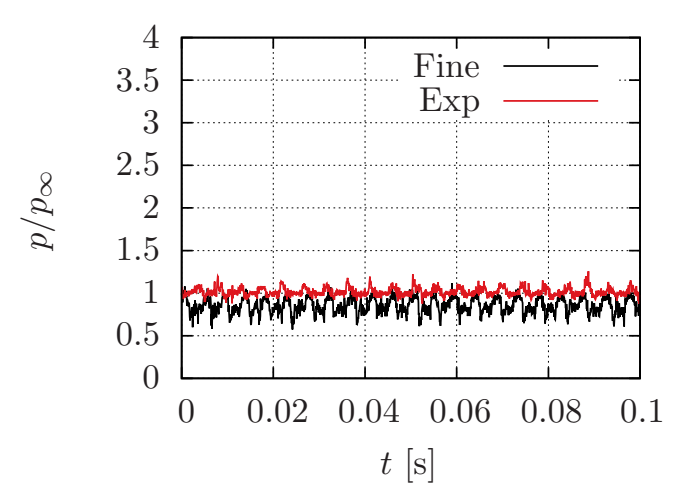

(g) Point 4, low actuation pressure

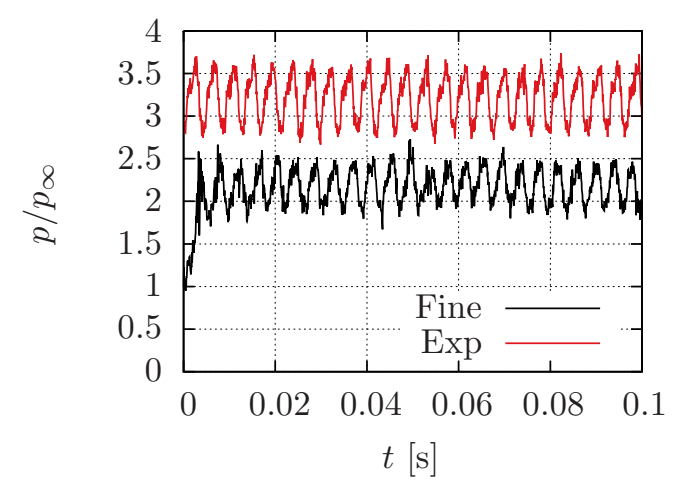

(b) Point 1, high actuation pressure

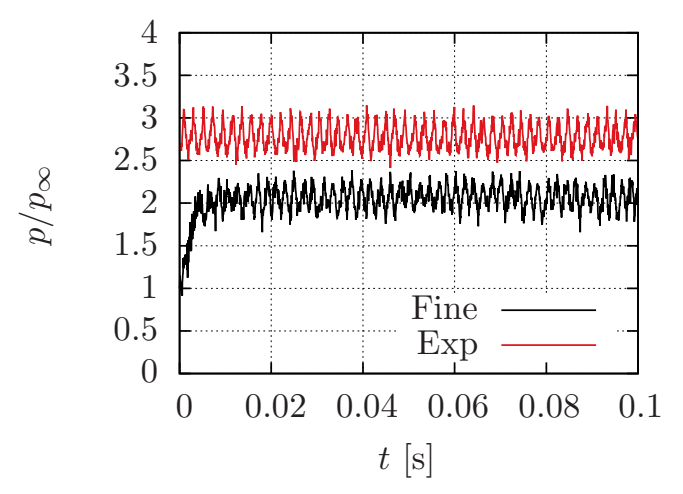

(d) Point 2, high actuation pressure

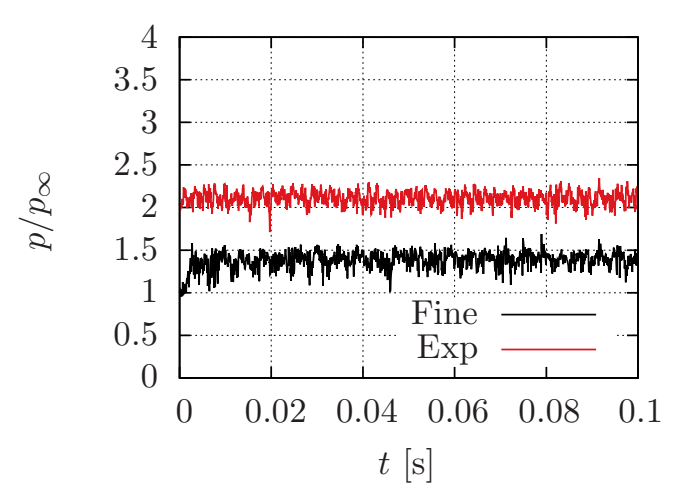

(f) Point 3, high actuation pressure

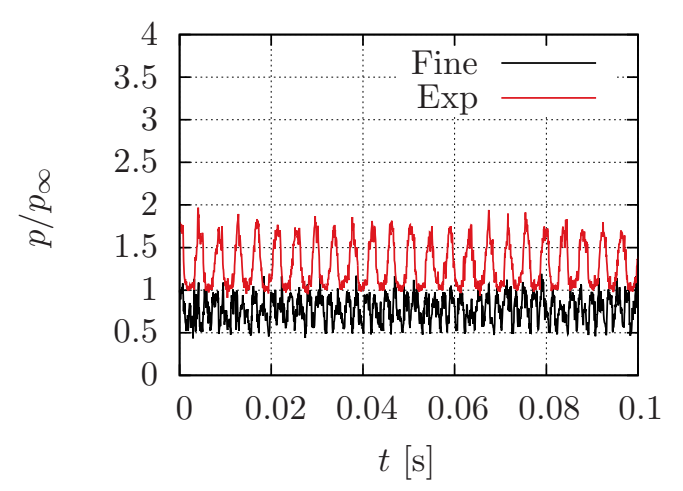

(h) Point 4, high actuation pressure

Figure 7. Pressure histories extracted from fine simulations 


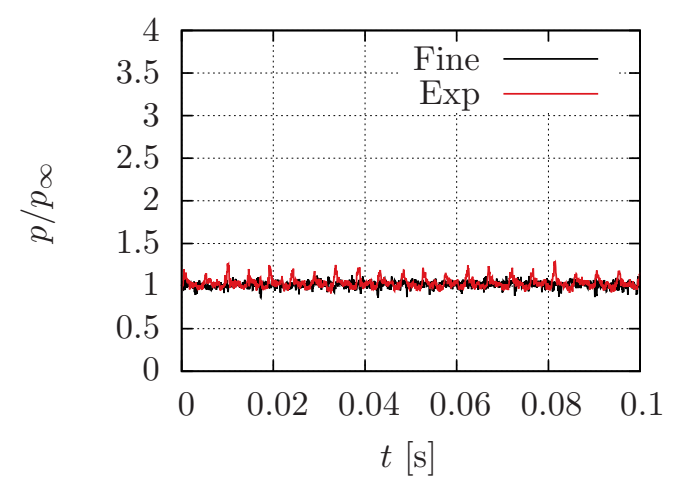

(a) Point 5, low actuation pressure

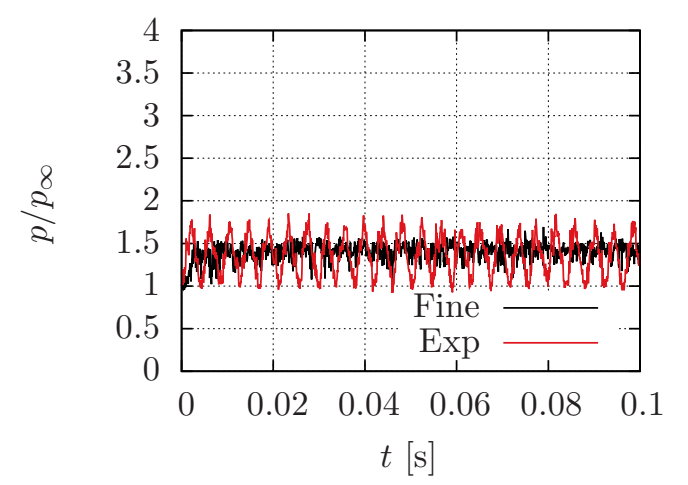

(b) Point 5, high actuation pressure

Figure 8. Pressure histories extracted from fine simulations, cont.

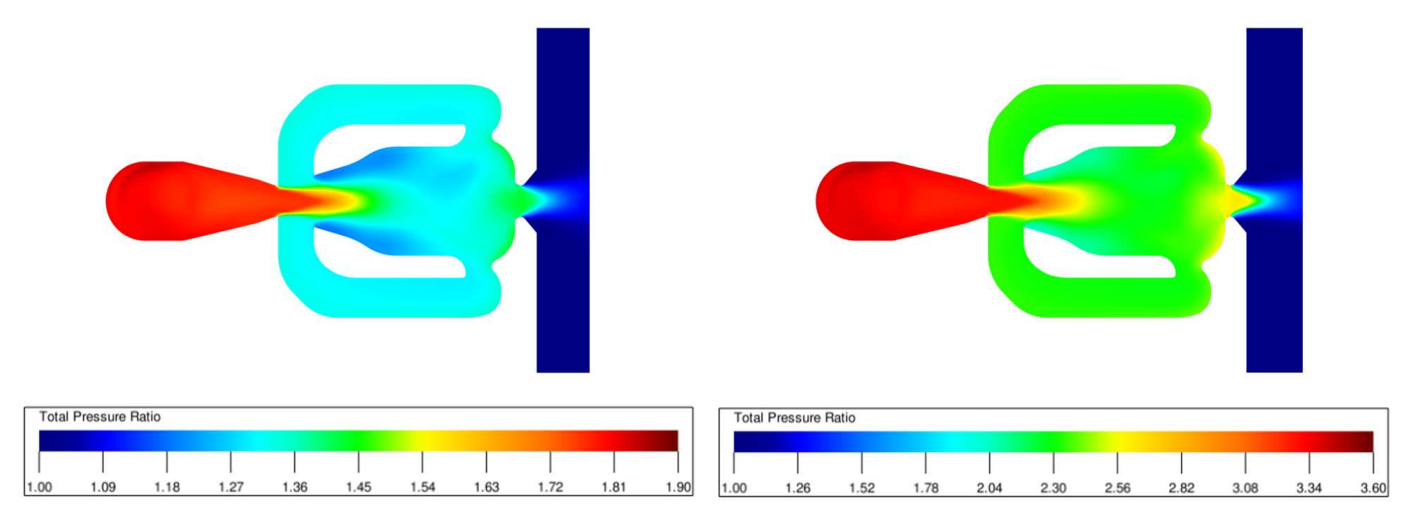

Figure 9. Total pressure contours inside the actuator for low (left) and high (right) actuation pressure 


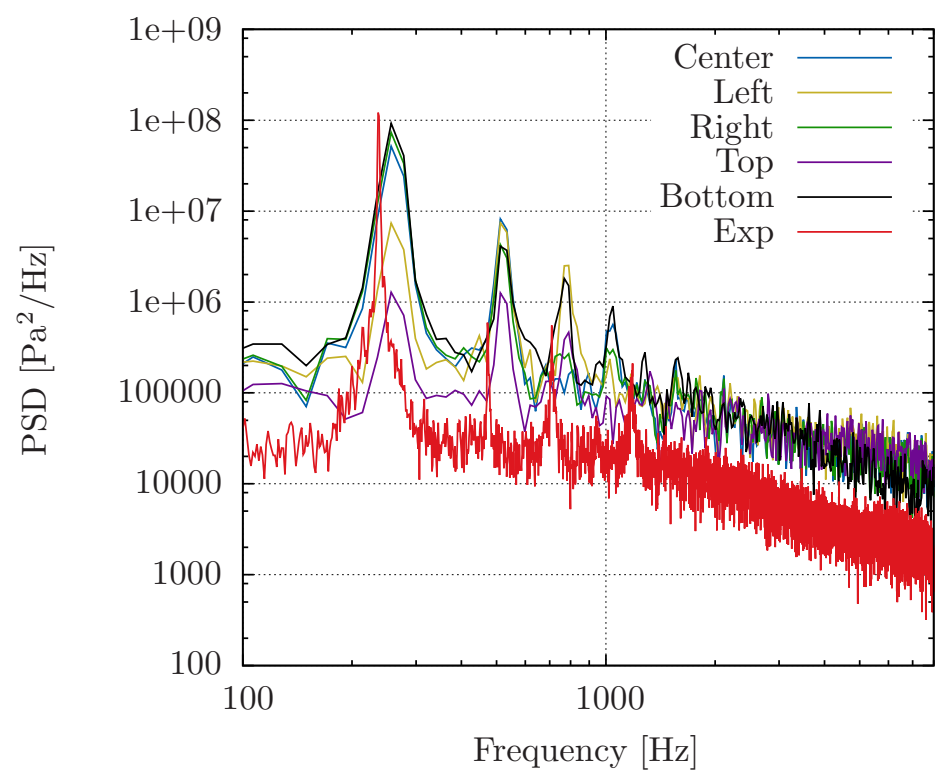

(a) Point 4, low actuation pressure

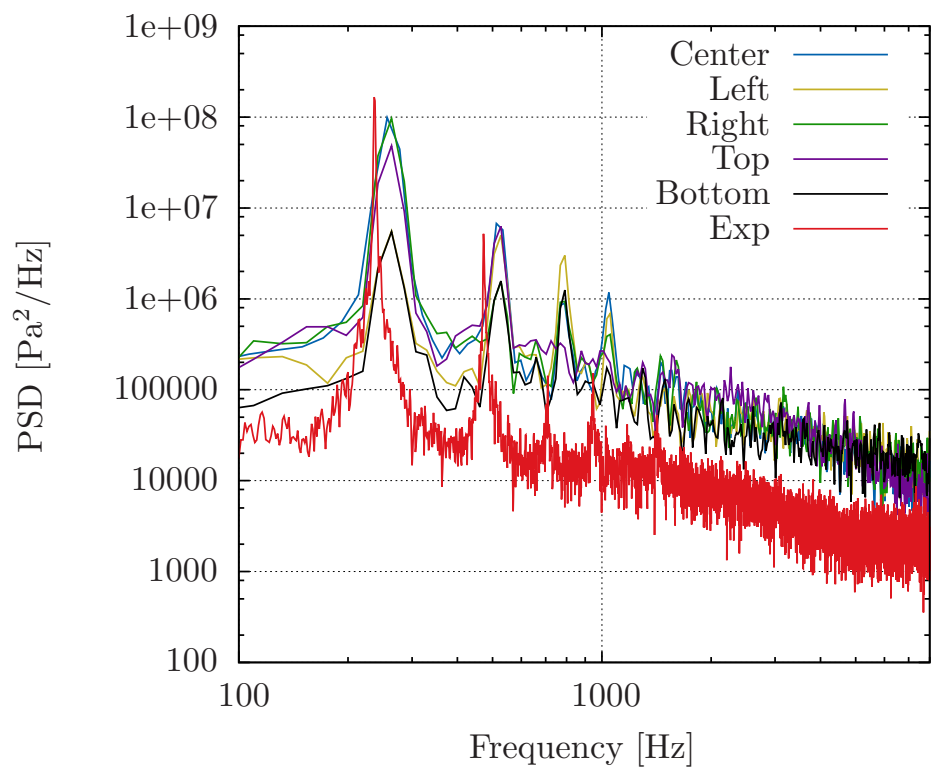

(b) Point 5, high actuation pressure

Figure 10. Sensitivity study: power spectral densities extracted from fine simulation 


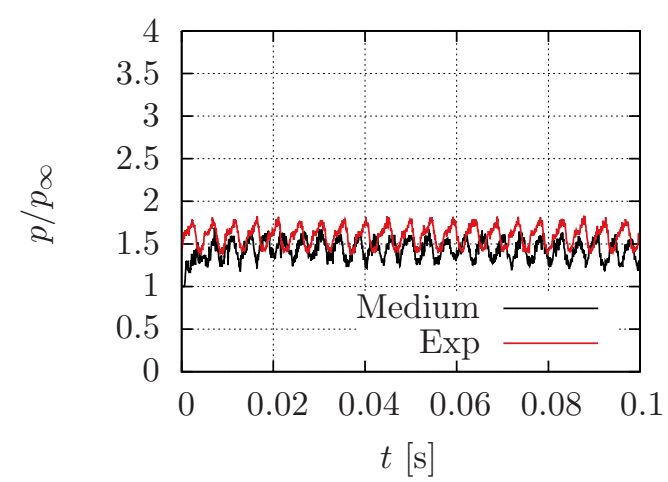

(a) Point 1, low actuation pressure

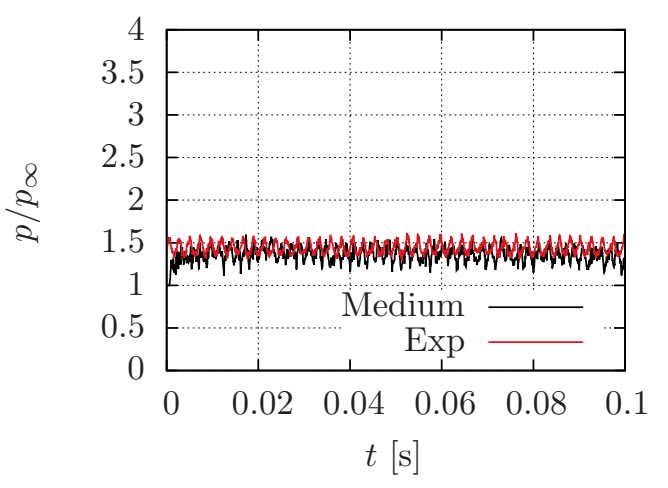

(c) Point 2, low actuation pressure

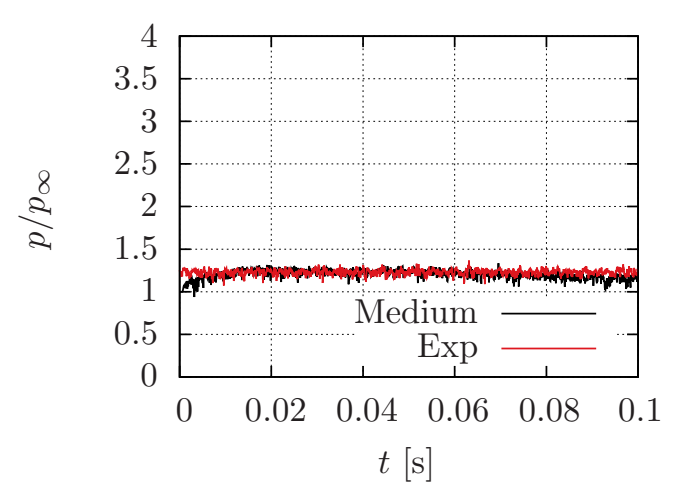

(e) Point 3, low actuation pressure

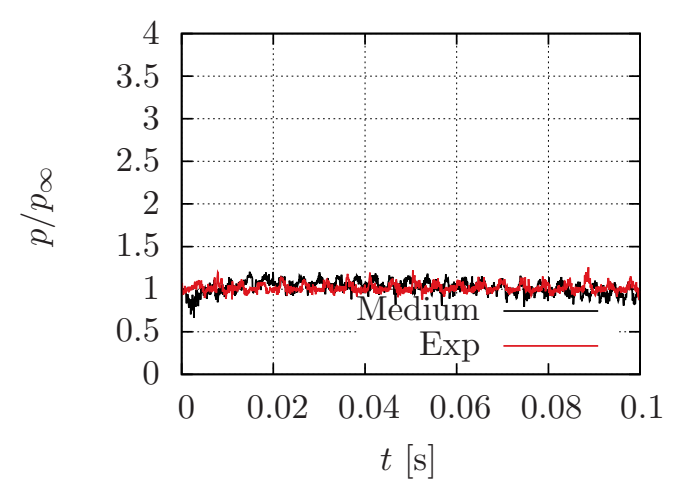

(g) Point 4, low actuation pressure

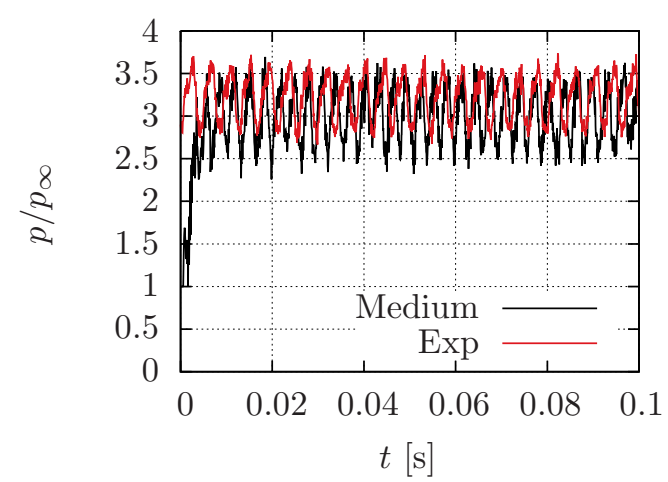

(b) Point 1, high actuation pressure

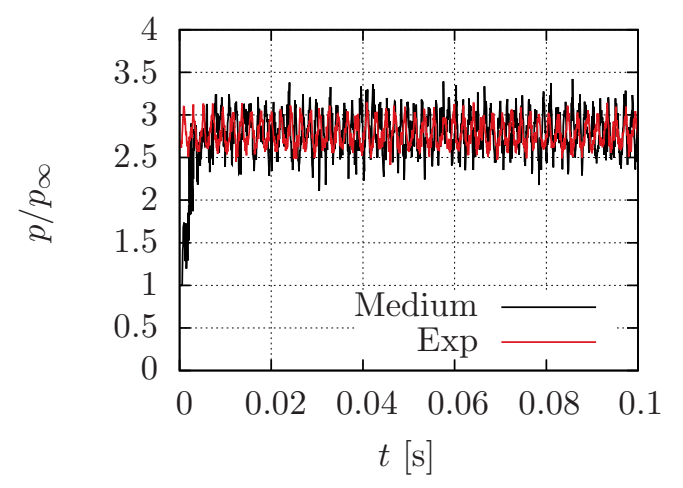

(d) Point 2, high actuation pressure

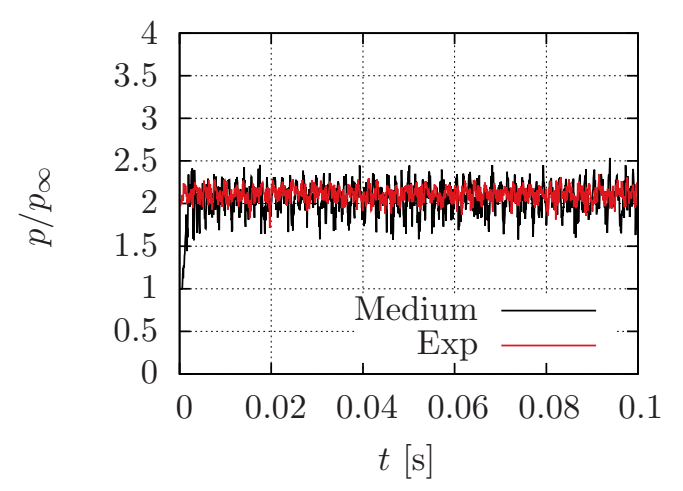

(f) Point 3, high actuation pressure

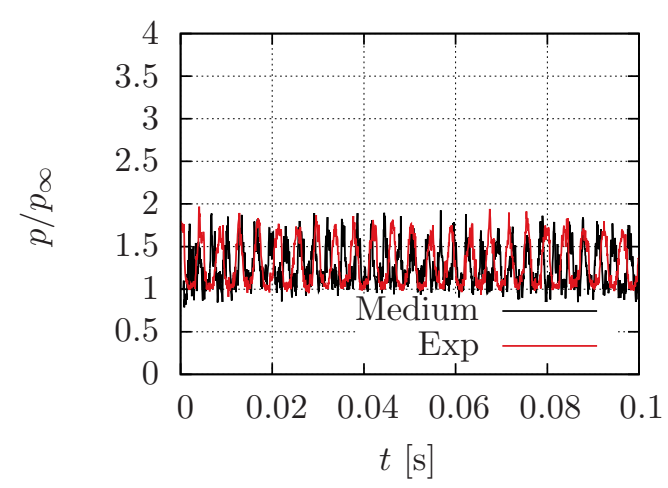

(h) Point 4, high actuation pressure

Figure 11. Pressure histories extracted from fine simulations with adjusted pressure ratio 


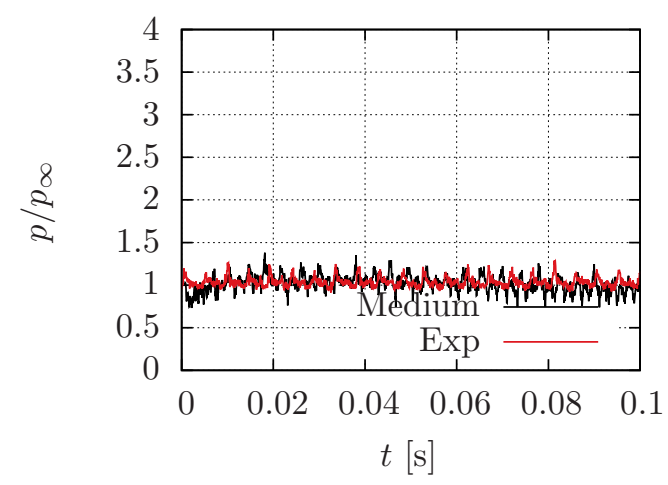

(a) Point 5, low actuation pressure

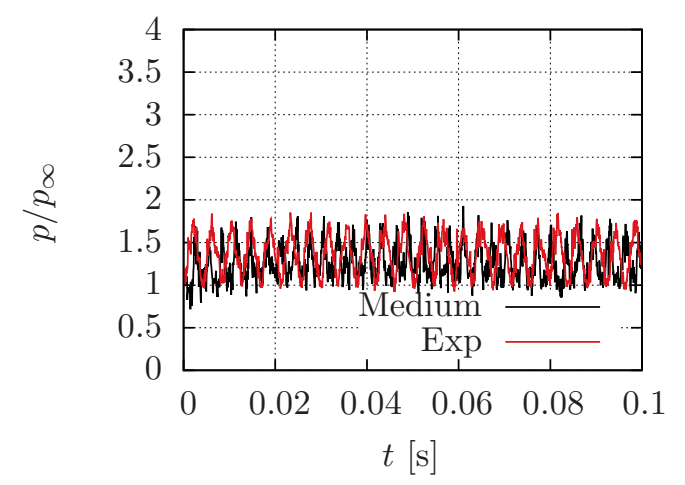

(b) Point 5, high actuation pressure

Figure 12. Pressure histories extracted from fine simulations with adjusted pressure ratio, cont. 

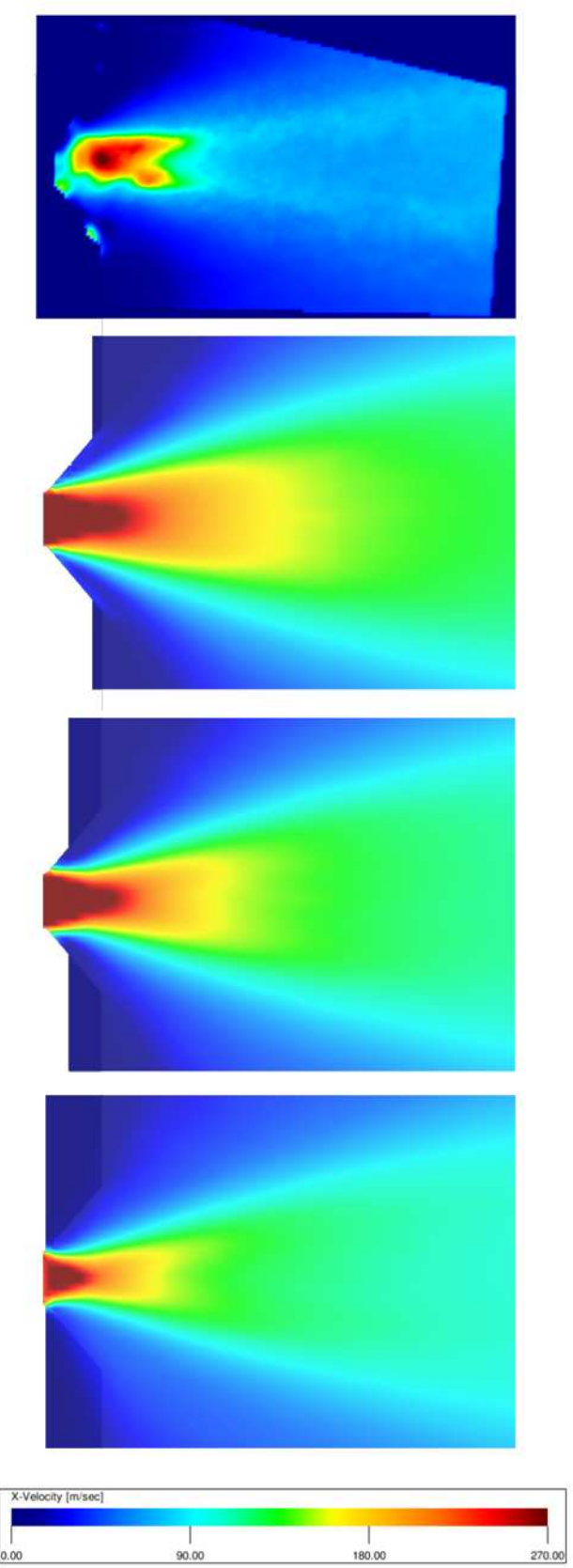

(a)
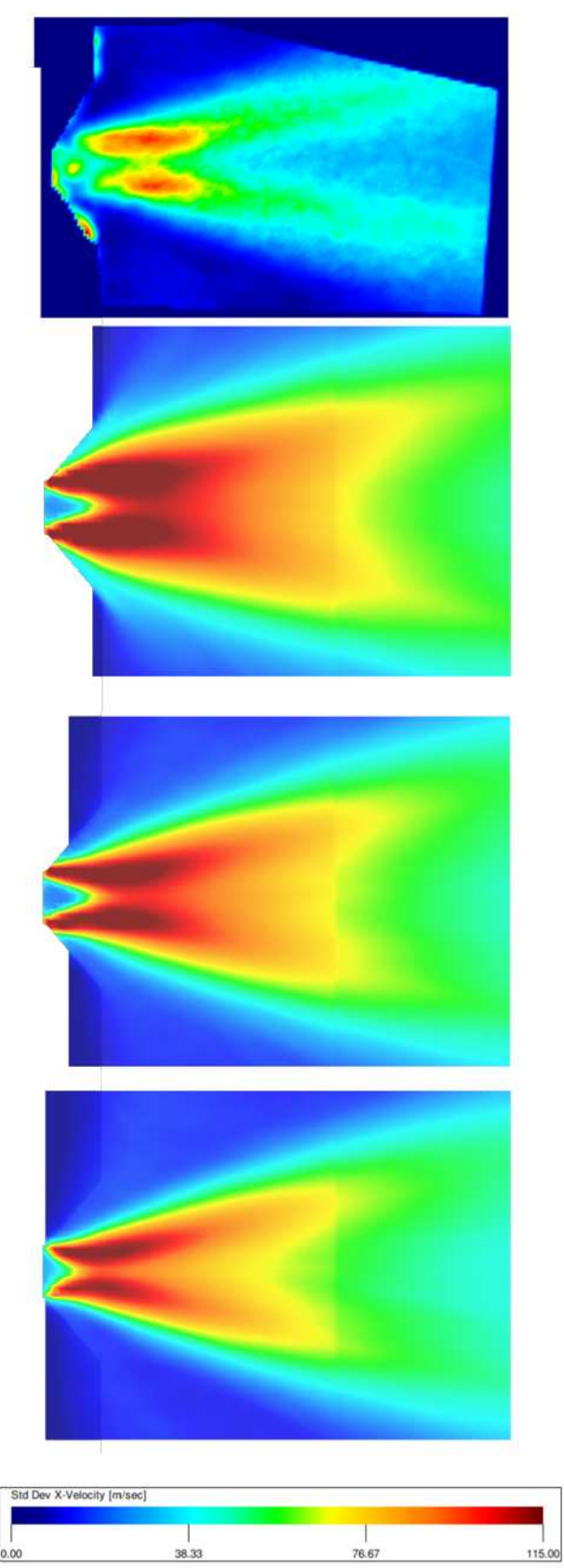

(b)

Figure 13. Mean and root mean square values of $x$-velocity 


\section{References}

${ }^{1}$ Kushner, L., Heineck, J., Storms, B., and Childs, R., "Visualization of a sweeping jet by laser speckle retro-reflective background oriented schlieren," 53rd AIAA aerospace sciences meeting, Kissimmee, Vol. 2015-1690, 2015.

${ }^{2}$ Koklu, M. and Melton, L., "Sweeping Jet Actuator in a Quiescent Environment," AIAA paper, Vol. 2013-2477, 2013.

${ }^{3}$ Vatsa, V. N., Kiklu, M., Wygnanski, I. L., and Fares, E., "Numerical Simulation of Fluidic Actuators for Flow Control Applications," 6th AIAA Flow Control Conference, Vol. 2012-3239, 2012.

${ }^{4}$ Vatsa, V. N., Casalino, D., Lin, J. C., and Appelbaum, J., "Numerical Simulation of a High Lift Configuration with Embedded Fluidic Actuators," 32nd AIAA Aplied Aerodynamics Conference, Vol. 2014-2142, 2014.

${ }^{5}$ Chen, H., Chen, S., and Matthaeus, W., "Recovery of the Navier-Stokes Equations Using a Lattice-Gas Boltzmann Method," Physical Review A, Vol. 45, No. 8, 1992, pp. 5339-5342.

${ }^{6}$ Qian, Y., d'Humieres, D., and Lallemand, P., "Lattice BGK Models for the Navier-Stokes Equation," Europhysics Letters, Vol. 17, 1992, pp. 479-484.

${ }^{7}$ Fares, E., Wessels, M., Li, Y., Gopalakrishnan, P., Zhang, R., Sun, C., Gopalaswamy, N., Roberts, P., Hoch, J., and Chen, H., "Validation of a Lattice-Boltzmann Approach for Transonic and Supersonic Flow Simulations," AIAA Journal, Vol. 2014-0952, 2014.

${ }^{8}$ Chen, H. and Teixeira, C. M., "H-Theorem and Origins of Instability in Thermal Lattice Boltzmann Models," Computer Physics Communications, Vol. 129, 2000, pp. 21-31.

${ }^{9}$ Chen, H., Teixeira, C. M., and Molvig, K., "Digital physics approach to computational fluid dynamics: some basic theoretical features," International Journal of Modern Physics C, Vol. 8, No. 4, 1997, pp. 675-684.

${ }^{10}$ Chen, H., Teixeira, C., and Molvig, K., "Realization of Fluid Boundary Conditions via Discrete Boltzmann Dynamics," International Journal of Modern Physics C, Vol. 9, No. 8, 1998, pp. 1281-1292.

${ }^{11}$ Chen, H., Kandasamy, S., Orszag, S. A., Succi, S., and Yakhot, V., "Extended Boltzmann Kinetic Equation for Turbulent Flows," Science, Vol. 301, No. 5633, 2003, pp. 633-636.

${ }^{12}$ Shan, X., Yuan, X.-F., and Chen, H., "Kinetic Theory Representation of Hydrodynamics: a Way Beyond the NavierStokes Equation," Journal of Fluid Mechanics, Vol. 550, 2006, pp. 413-441.

${ }^{13}$ Teixeira, C. M., "Incorporating Turbulence Models into the Lattice-Boltzmann Method," International Journal of Modern Physics C, Vol. 9, 1998, pp. 1159-1175.

${ }^{14}$ Brès, G. A., Freed, D. M., Wessels, M., Noelting, S., and Pérot, F., "Flow and Noise Predictions for the Tandem Cylinder Aeroacoustic Benchmark," Physics of Fluids, Vol. 24, No. 3, March 2012, pp. 036101.

${ }^{15}$ Khorrami, M. R., Fares, E., and Casalino, D., "Towards Full Aircraft Airframe Noise Prediction: Lattice Boltzmann Simulations," AIAA Paper 2014-xxxx, June 2014.

${ }^{16}$ Li, Y., Shock, R., Zhang, R., and Chen, H., "Numerical Study of Flow Past an Impulsively Started Cylinder by Lattice Boltzmann Method," Journal of Fluid Mechanics, Vol. 519, 2004, pp. 273-300.

${ }^{17}$ Fares, E., Jelic, S., Kuthada, T., and Schroeck, D., "Lattice Boltzmann Thermal Flow Simulation and Measurements of a modified SAE Model with Heated Plug," Proceedings of FEDSM2006-98467, 2006.

${ }^{18}$ Fares, E., "Unsteady Flow Simulation of the Ahmed Reference Body using a Lattice Boltzmann Approach," Journal of Computers and Fluids, Vol. 35, 2006, pp. 940-950.

${ }^{19}$ Casalino, D., Ribeiro, A. F. P., Fares, E., and Nölting, S., "Lattice-Boltzmann Aeroacoustic Analysis of the LAGOON Landing Gear Configuration," AIAA Journal, Vol. 52, No. 6, 2014, pp. 1232-1248. 